\title{
Primulina cardaminifolia (Gesneriaceae), a rare new species from limestone areas in Guangxi, China
}

\author{
Wei-Bin Xu', Yan Liu', Yoshiko Kono ${ }^{2}$, Hsuan Chang ${ }^{3}$, Ching-l Peng ${ }^{2^{*}}$ and Kuo-Fang Chung ${ }^{3^{*}}$
}

\begin{abstract}
Background: Primulina cardaminifolia Yan Liu \& W.B. Xu (Gesneriaceae), a distinct new species with imparipinnate leaves, is described and illustrated from a limestone valley in Guangxi Zhuangzu Autonomous Region, China. To assure its generic placement and phylogenetic affinity, phylogenetic analyses were performed using DNA sequences of nuclear ITS and chloroplast trnL-F intron spacer region. Additionally, somatic chromosome number was counted and pollen stainability was tested.
\end{abstract}

Results: Phylogenetic analyses support its placement in Primulina; however, two phylogenetically distinct ITS sequence types were detected, suggesting a probable hybrid origin. Its pollen stainability is $100 \%$ and its chromosome number, $2 n=36$, is congruent with all known counts of diploid species of the genus.

Conclusion: All available data support the recognition of the new species Primulina cardaminifolia and suggest that it could have derived from homoploid hybrid speciation. Color plates, line drawings and a distribution map are provided to aid in identification.

Keywords: Chromosome number; Flora of China; Homoploid hybrid; Sino-Vietnamese limestone karst; Molecular taxonomy; Primulina pinnata

\section{Background}

Recent progress in molecular phylogenetic studies of the Old World Gesneriaceae has foreseen the restructuring of the highly heterogeneous Chirita D. Don ( $\mathrm{Li}$ and Wang 2007; Möller et al. 2009; Möller et al. 2011). However it was unexpected that Chirita has been forsaken (Wang et al. 2011; Weber et al. 2011) and the formerly monotypic Primulina Hance recircumscribed and expanded to include Chirita sect. Gibbosaccus C.B. Clarke, Chiritopsis W.T. Wang, and two species of Wentsaiboea D. Fang \& D.H. Qin (Wang et al. 2011; Weber et al. 2011). With over 130 species transferred to (Wang et al. 2011; Weber et al. 2011; Xu et al. 2012b) and more than ten species newly described (Liu et al. 2011; Hong et al. 2012; Huang et al. 2012; Li et al. 2012; Wen et al. 2012a, b, c; Wu et al. 2012a, 2012b; Xu et al. 2012a; Chung et al. 2013), Primulina has now become one of the largest

\footnotetext{
* Correspondence: bopeng@sinica.edu.tw; kuofangchung@ntu.edu.tw ${ }^{2}$ Herbarium (HAST), Biodiversity Research Center, Academia Sinica, Nangang, Taipei 115, Taiwan

${ }^{3}$ School of Forestry and Resource Conservation, National Taiwan University, Daan, Taipei 106, Taiwan

Full list of author information is available at the end of the article
}

genera of the Old World Didymocarpoid Gesneriaceae. Under this new delimitation, Primulina is essentially a calciphilous genus distributed in southern China and adjacent northern Vietnam, with the major center of diversity in the limestone karsts of Guangxi Zhuangzu Autonomous Region (Wang et al. 1998; Li and Wang 2004; Hou et al. 2010; Wei 2010; Weber et al. 2011; Xu et al. 2012a, b).

Although the new generic circumscription reflects better the evolutionary relationships and ecological preferences of the genus, diagnostic characters of Primulina, such as perennial habit and acaulescent rosette (Weber et al. 2011) and the basic chromosome number, $x=18$ (Christie et al. 2012; Liu et al. 2012), are not exclusive to the genus. The lack of strong morphological synapomorphies to distinguish it from related genera in the region necessitates molecular data in ascertaining generic placements (Xu et al. 2012a, b). For example, the transfer of Chirita tamiana B.L. Burtt to Primulina [i.e., P. tamiana (B.L. Burtt) Mich. Möller \& A. Weber] made without support from molecular data by Weber et al. (2011) was recently challenged by chromosomal cytology and molecular data (Christie et al. 2012). Similarly, our ongoing molecular 
phylogenetic studies have also identified a number of misplacments in Primulina (Chung, unpubl. data).

In the course of a floristic survey in central Guangxi in 2007, a distinct species of Gesneriaceae with imparipinnate leaves was collected by the authors. After consulting national and local floras and the relevant literature (Wang et al. 1998; Li and Wang 2004; Wei 2010; Liu et al. 2011; Wang et al. 2011; Wu et al. 2012a; Xu et al. 2012a, b) as well as herbarium specimens, we conclude that it represents a new species of Primulina, which is described and illustrated here. Chromosome count of the new species is in agreement with those reported for
Primulina in Christie et al. (2012). Its generic placement is further confirmed by molecular and chromosome data.

\section{Methods}

\section{Chromosome preparations}

The plant for chromosome studies was collected from the type locality and cultivated in the experimental greenhouse of Academia Sinica, Taipei. A voucher specimen (Ku et al. 2035) has been deposited in HAST. Root tips were gathered and pretreated in $2 \mathrm{mM}$ 8-hydroxyquinoline at $15-18^{\circ} \mathrm{C}$ for about $6 \mathrm{~h}$ and fixed overnight in an ethanol-acetic acid solution $(3: 1)$ below $4^{\circ} \mathrm{C}$. The

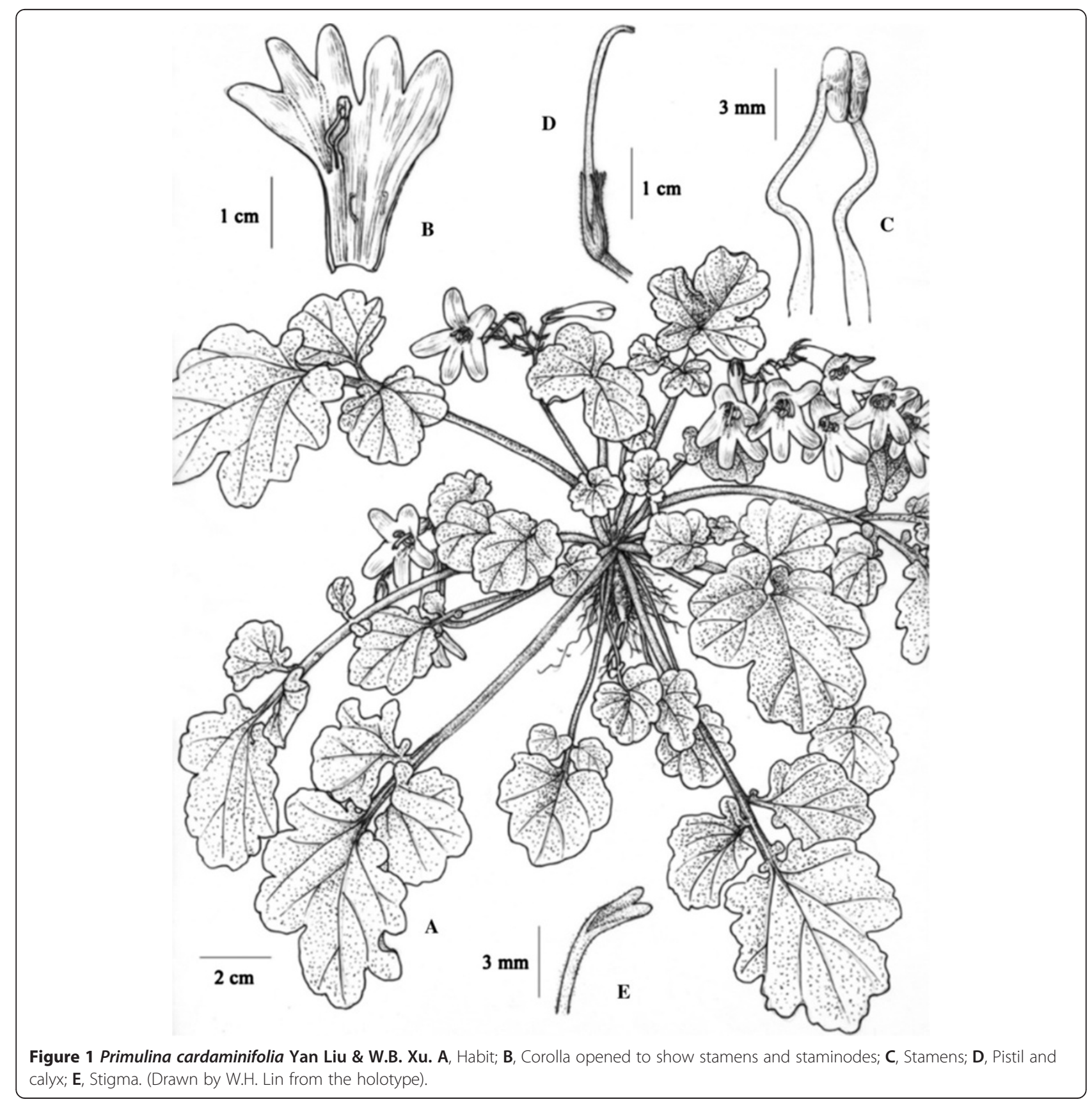


chromosomes were stained and macerated in $2 \%$ acetic orcein with $1 \mathrm{~N}$ hydrochloric acid (10:1). Classification of chromosome complement based on centromere position at mitotic metaphase followed Levan et al. (1964).

\section{Molecular methods}

DNA sequences of the nuclear ribosomal internal transcribed spacers (ITS) and the chloroplast trnL-F intron spacer region were gathered using protocols outlined in $\mathrm{Xu}$ et al. (2012a). Because direct sequencing of the ITS PCR products of the new species resulted in DNA sequences with overlapping signals, molecular cloning was performed. Following the manufacturer's protocol, the purified ITS templates were ligated to the pGEMT-T vector system (Promega, Madison, Wisconsin, USA) and subsequently transformed into competent cells (DH5 $\alpha$ ) to perform molecular cloning. After overnight culture at $37^{\circ} \mathrm{C}$ on the LB ampicillin/IPTG/X-gal selective plate, colonies carrying the ITS insert were identified by color (white) and further verified by PCR using the T7 and SP6 promoter primer pairs (Promega, Madison, Wisconsin, USA). Ten colonies with positive ITS insert were then transferred and grown in $2 \mu \mathrm{LB}$ medium at $37^{\circ} \mathrm{C}$ for $15 \mathrm{~h}$. Plasmids were extracted using the Mini
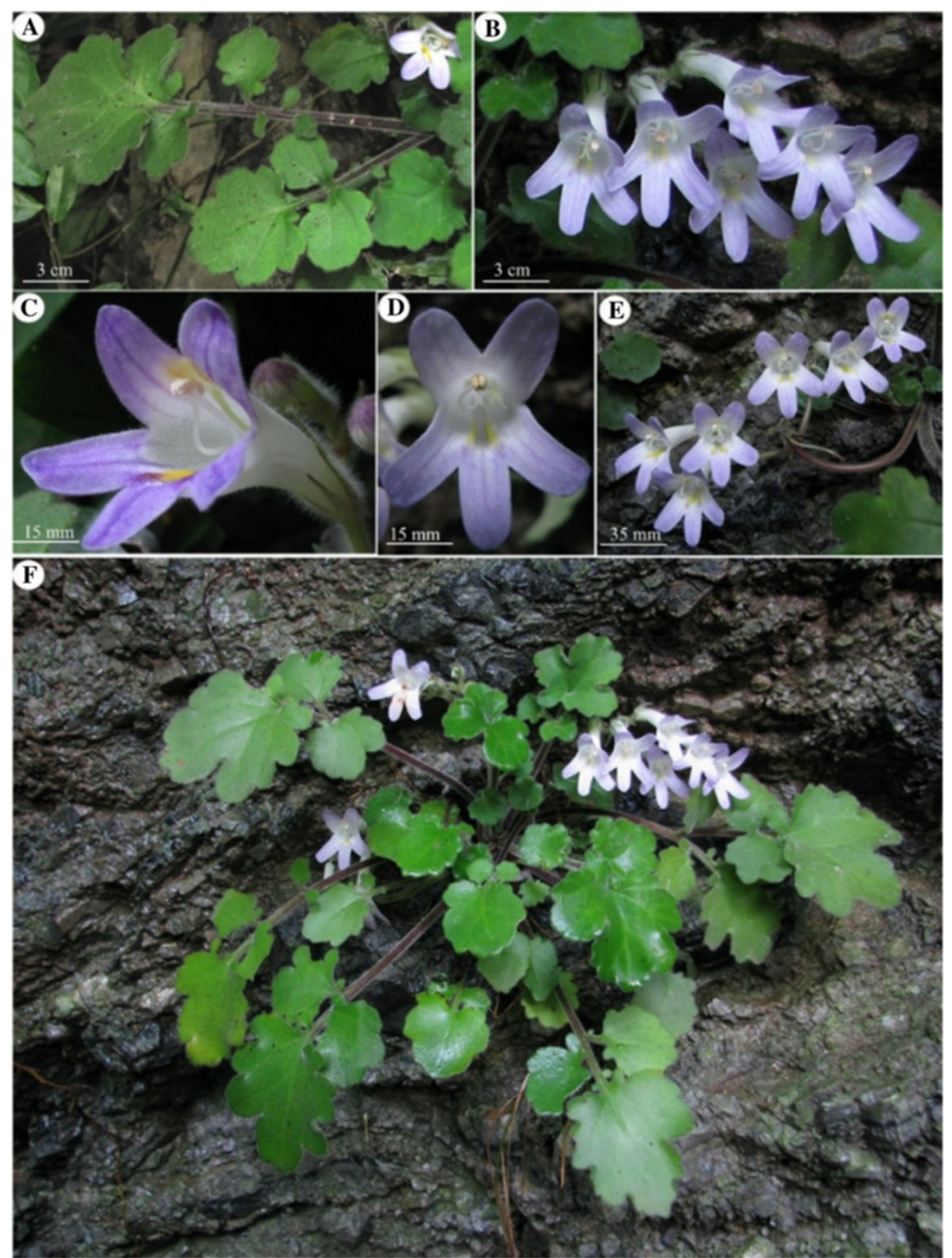

Figure 2 Primulina cardaminifolia Yan Liu \& W.B. Xu. A, Leaves; B, Flowers; C, Flower side view; D, Flower face view; E, Cyme; F, Habit. 
Plus ${ }^{\text {Th }}$ Plasmid DNA Extraction System (Viogene, Taipei, Taiwan) and cycle-sequenced using the T7 and SP6 promoter primer pairs.

For phylogenetic analyses, matrices of Xu et al. (2012a) were adopted, keeping one accession for each species (Appendix 1) and ITS and trnL-F regions as separated matrices. Primulina pinnata (W.T. Wang) YinZ. Wang, which was not included in Xu et al. (2012a) because of the presence of an access of ambiguous sites in its ITS sequence in the GenBank (i.e., FJ501349), were added in current analyses to test for its putative relationships with the new species. The final matrix contained 24 species of Primulina with Petrocodon dealbatus Hance, Pet. scopulorum (Chun) YinZ. Wang, and Didymocarpus podocarpus C.B. Clarke chosen as outgroups based on recent phylogenetic analyses (Möller et al. 2011; Weber et al. 2011). The DNA sequences were aligned using the program MUSCLE implemented in the software MEGA5 (Tamura et al. 2011) with minor manual adjustments. Phylogenetic trees were reconstructed separately for ITS and trnL- $F$ based on maximum parsimony (MP) and maximum likelihood (ML) criteria implemented in MEGA5. MP trees were searched using the Tree-Bisection-Reconnection (TBR) search option with the initial trees setting at 50, MP search level setting at 5 , and maximum number of trees setting at 2000. Clade supports were calculated based on 100 bootstrap resamplings (parsimony bootstrap; $\mathrm{PB})$. ML trees were reconstructed using the nearestneighbor-interchange (NNI) method with all site used and the initial tree automatically selected under the model(s) selected by MEGA5. Clade supports of ML analysis were evaluated based on 100 bootstrap resamplings (likelihood bootstrap; LB).

\section{Results and discussion}

Taxonomic treatment

\section{Primulina cardaminifolia}

Yan Liu \& W.B. Xu, sp. nov.-TYPE: CHINA. Guangxi Zhuangzu Autonomous Region, Laibin Shi (City), Fenghuang Zhen (Township), alt. $280 \mathrm{~m}$, on moist limestone rock face in a valley, 14 July 2008, Wei-Bin $\mathrm{Xu}$ \& Yan Liu 08050 (holotype: IBK; isotypes: HAST and PE). 碎米薺葉報春苣苔 Figures 1, 2.

\section{Diagnosis}

Primulina cardaminifolia Yan Liu \& W.B. Xu resembles Primulina pinnata (W.T. Wang) YinZ. Wang in having imparipinnate leaves, but is clearly distinct from this species by the ovate-cordate terminal leaflet of 3-7 $\times 3$ $6.5 \mathrm{~cm}, 1$ or 2 pairs of broadly ovate to sub-ovate lateral leaflets, 1-3-branched cymes with 3 to 10-flowers, and the entire-margined calyx lobes with acuminate apex.

\section{Description}

Herbs perennial. Rhizome subterete, 3-5 $\mathrm{mm}$ across. Leaves 5-7, in basal rosette, imparipinnate, $10-20 \mathrm{~cm}$ long, papery when dry; petiole subterete, $6-12.5 \mathrm{~cm}$ long, densely pubescent; terminal leaflet ovate-cordate, 3-7 $\times 3-6.5 \mathrm{~cm}$, apex obtuse, base cordate, margin repand to irregularly pinnately lobed, densely pubescent on both

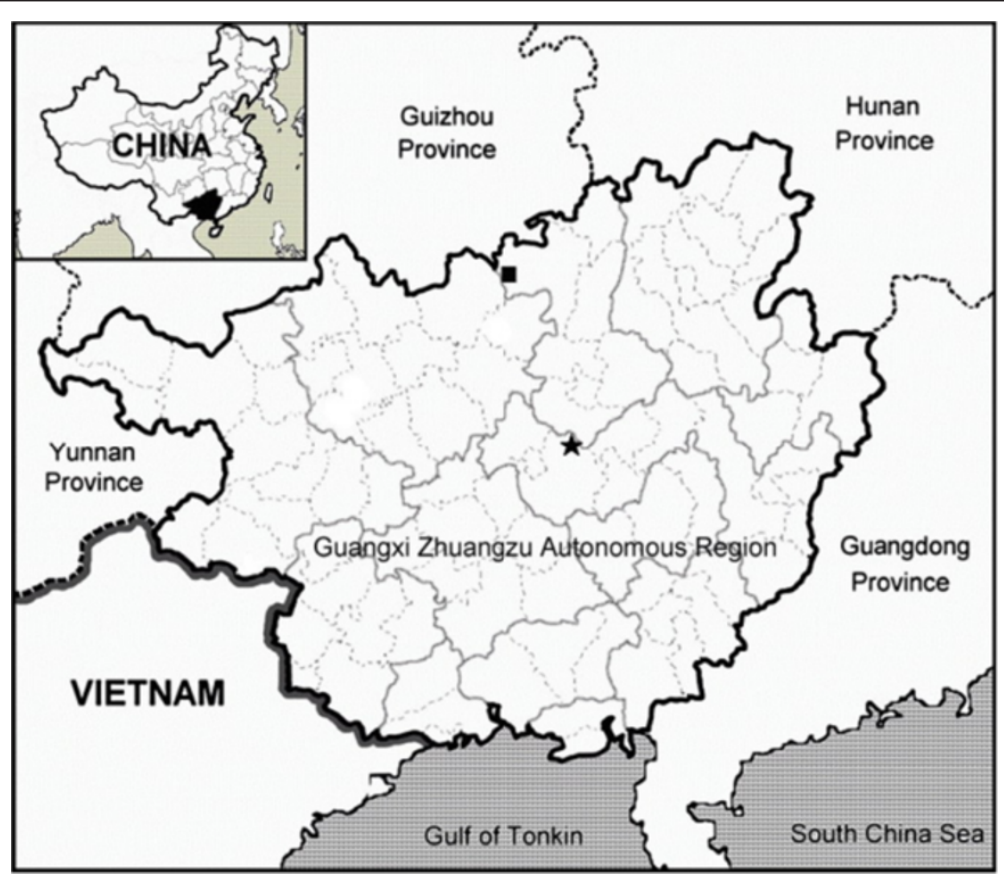

Figure 3 Distribution of Primulina cardaminifolia ( $\star$ ) and P. pinnata ( $\square$ ) in Guangxi Zhuangzu Autonomous Region, China. 
surfaces; lateral leaflets 1 or 2 pairs, opposite or alternate, broadly ovate to rotund, $1-3 \times 1-3 \mathrm{~cm}$, margin repand to irregularly pinnately lobed, densely pubescent on both surfaces, petiolules short, 2-10 mm long. Cymes 2-4, axillary, 1-3-branched, 3-10-flowered; peduncle 4-8 cm long, 1-2 $\mathrm{mm}$ in diam., densely pubescent; bracts 2 , opposite, linear-lanceolate, $7-8 \times 2-3 \mathrm{~mm}$, margin entire, pubescent; pedicel 4-7 mm long, densely pubescent. Calyx 5-parted to base, lobes lanceolate-linear, $8-12 \times 2-3 \mathrm{~mm}$, apex acuminate, outside pubescent, inside glabrous, margins entire. Corolla white to pale purple, 3.2-3.5 cm long, outside glandular-pubescent, inside sparsely puberulent, with 2 pale-yellow stripes; corolla tube $2.1-2.3 \mathrm{~cm}$ long, $8-12 \mathrm{~mm}$ in diam. at the mouth, $2.5-3 \mathrm{~mm}$ in diam. at the base; limb pale purple, distinctly 2-lipped; adaxial lip 2-parted to over the middle, lobes oblong, 8-10 × 6-7 mm; abaxial lip 3-lobed to over the middle, lobes oblong, $12-13 \times 4-5 \mathrm{~mm}$; stamens 2 , adnate to $1.5 \mathrm{~cm}$ above the corolla tube base; filaments linear, ca. $1.2 \mathrm{~cm}$ long, geniculate above the base, sparsely glandular-puberulent; anthers ca. $3 \mathrm{~mm}$ long, ca. $1.5 \mathrm{~mm}$ wide, dorsifixed, glabrous; staminodes 2, ca. $5 \mathrm{~mm}$ long, apex capitate, glabrous, adnate to ca. $6 \mathrm{~mm}$ above the base of corolla tube. Disc ring-like, ca. $1 \mathrm{~mm}$ in height, margin repand, glabrous. Pistil $2.5-2.8 \mathrm{~cm}$ long, ovary $7-8 \mathrm{~mm}$ long, ca. $1.5 \mathrm{~mm}$ across, puberulent; style $1.5-1.8 \mathrm{~cm}$ long, ca. $0.6 \mathrm{~mm}$ across, puberulent; stigma obtrapeziform, ca. 2 $\mathrm{mm}$ long, apex 2-lobed. Capsules not seen.

\section{Additional specimens examined}

CHINA. Guangxi Zhuangzu Autonomous Region, Laibin Shi, Fenghuang Zhen, 3 July 2008, Wei-Bin Xu \& Yan Liu 08040 (IBK); same locality, 8 Sep 2008, Wei-Bin Xu \& Kuo-Fang Chung 08472 (IBK), Shin-Ming Ku et al. 2035 (HAST); same locality, 28 June 2007, Hong-Jin Wei \& Wei-Bin Xu 07244 (IBK).

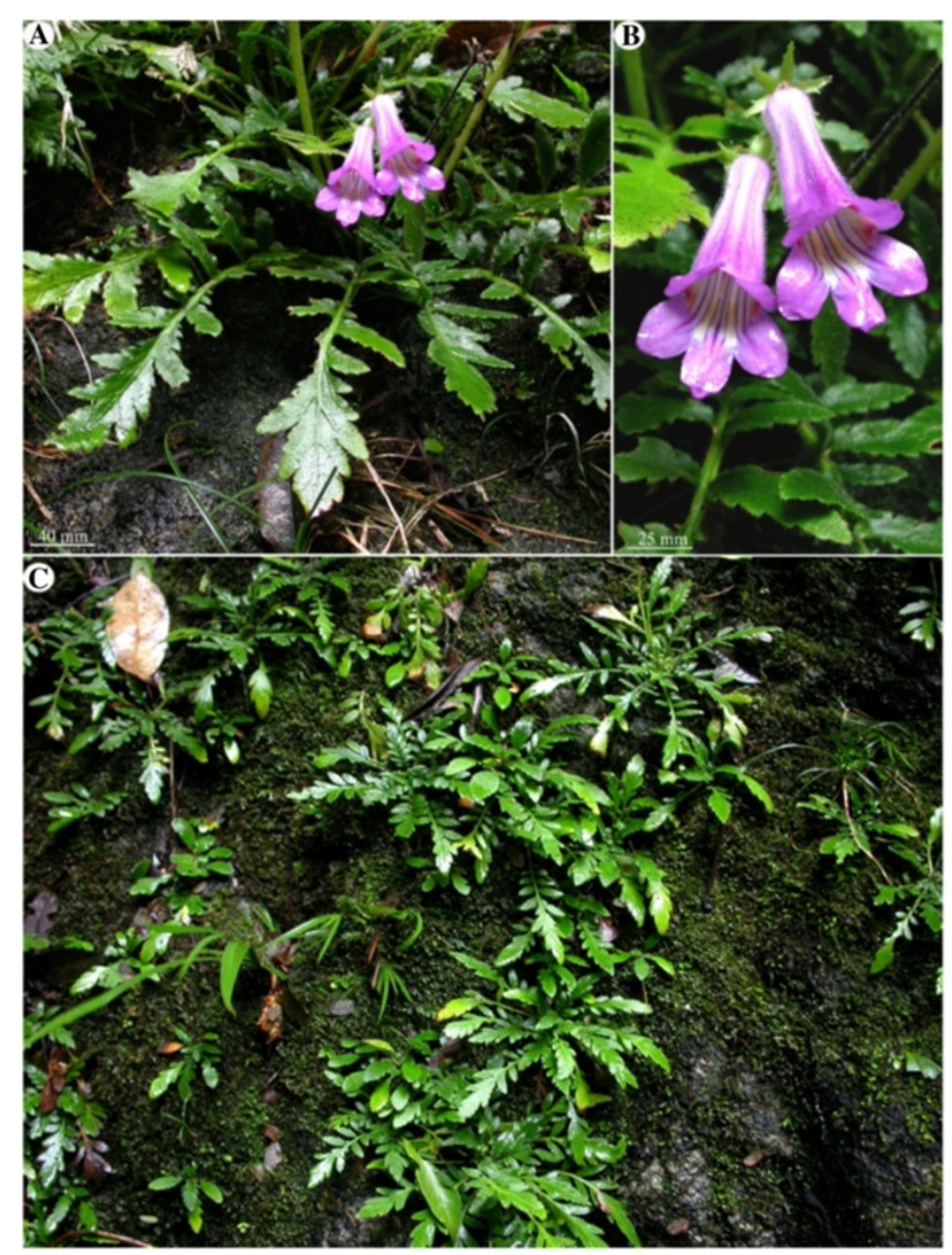

Figure 4 Primulina pinnata (W.T. Wang) YinZ. Wang. A, Habit; B, Flowers; C, Habitat. 


\section{Ecology and distribution}

Primulina cardaminifolia is extremely rare, currently known only from the type locality in Laibin Shi, Guangxi Zhuangzu Autonomous Region, China (Figure 3). It grows on a moist limestone rock face in a valley.

\section{Phenology}

Flowering from June to July; fruits not observed.

\section{Etymology}

The specific epithet is derived from the leaves resembling those of the genus Cardamine L. (Brassicaceae).

\section{Notes}

Primulina cardaminifolia resembles Primulina pinnata (W.T. Wang) YinZ. Wang (Figure 4), differing by the terminal leaflet being ovate-cordate, 3-7 × 3-6.5 cm (vs. oblong, $1.6-4.5 \times 0.7-2.5 \mathrm{~cm}$ ); the lateral leaflets 1 or 2 pairs, broadly ovate to rotund, $1-3 \times 1-3 \mathrm{~cm}$ (vs. $3-5$ pairs, oblong to oblong-lanceolate, $0.5-2.5 \times 0.4-1.5 \mathrm{~cm}$ ); cymes 1-3-branched, 3-10-flowered (vs. 1-branched, 1-3flowered); calyx lobe with acuminate apex and entire margins (vs. acute to obtuse at apex and margins denticulate).

\section{Chromosome cytology}

Somatic chromosomes at metaphase of Primulina carda minifolia were determined to be $2 n=36$ (Figure 5). The 36 chromosomes were small and gradually varied from ca. $0.6 \mu \mathrm{m}$ to $1.3 \mu \mathrm{m}$ in length. Most chromosomes had centromeres at median positions, while those of some of the shorter chromosomes could not be determined. Satellites were not observed.

In Primulina, chromosome numbers are uniformly diploid with $2 n=36$ except for $2 n=32$ in P. tamiana that was misplaced in the genus (Christie et al. 2012) and a polyploid with $2 n=72$ in P. longgangensis (W.T. Wang) YinZ. Wang (Christie et al. 2012; Liu et al. 2012; Yang et al. 2012). Chromosomes of Primulina at somatic metaphase are generally small, ranging from 0.7 to 1.6 $\mu \mathrm{m}$ (Christie et al. 2012; Liu et al. 2012), to which $P$. cardaminifolia agrees (Figure 5). Our chromosome count of $2 n=36$ in $P$. cardaminifolia agrees with basic chromosome number, $x=18$, and supports its generic placement in the genus.

\section{Phylogenetic analyses}

Results of molecular cloning of the ITS PCR product revealed two phylogenetically distinct ITS sequences (Figure 6A) with the length of 643 (P. cardaminifolia-A) and 634 (P. cardaminifolia-B) bp, respectively, while only one cpDNA sequence type was detected in the species. With the addition of these two ITS sequences, the ITS matrix contained 28 accessions of 675 aligned positions, of which 182 (26.96\%) were parsimoniously informative. Based on the Kimura 2-parameter model using a discrete Gamma distribution $(\mathrm{K} 2+\mathrm{G})$ with 5 rate categories selected by the corrected Akaike Information Criterion (AICc) implemented in MEGA5, a single ML tree (log
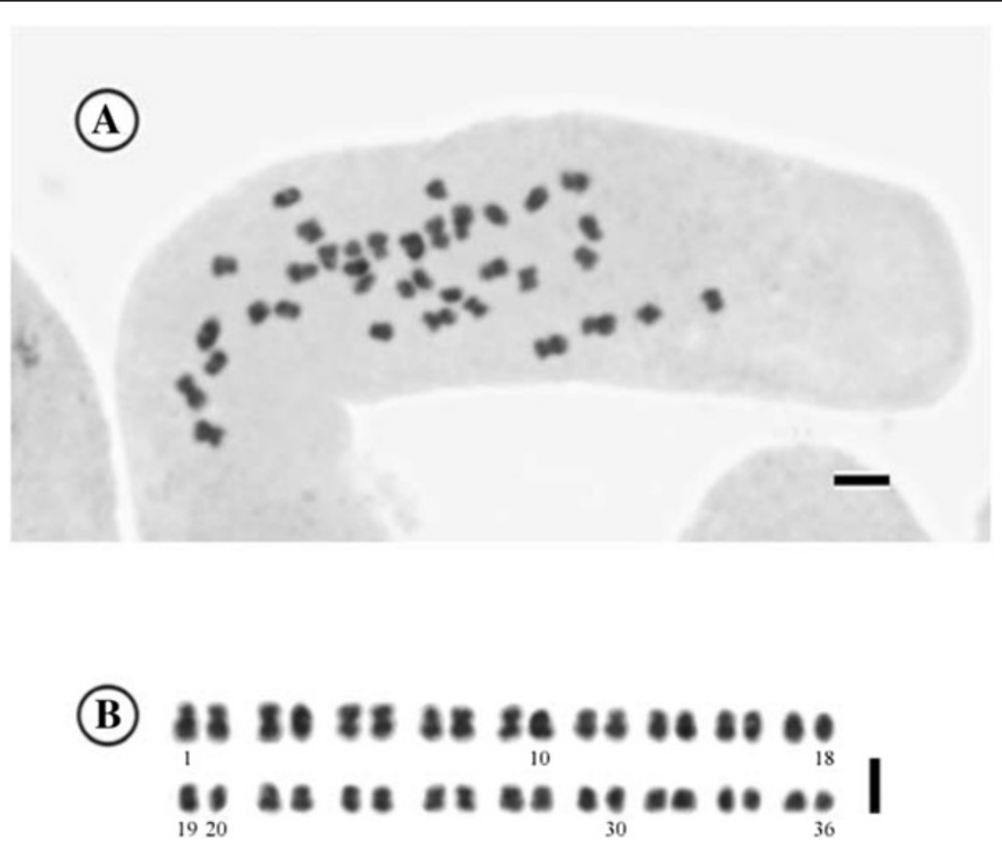

Figure 5 Somatic chromosomes at metaphase of Primulina cardaminifolia ( $2 n=36$, from Ku et al. 2035, HAST). A, Microphotograph; B Somatic chromosomes serially arranged by their chromosome length and the position of centromeres. Scale bars $=2 \mu \mathrm{m}$. 


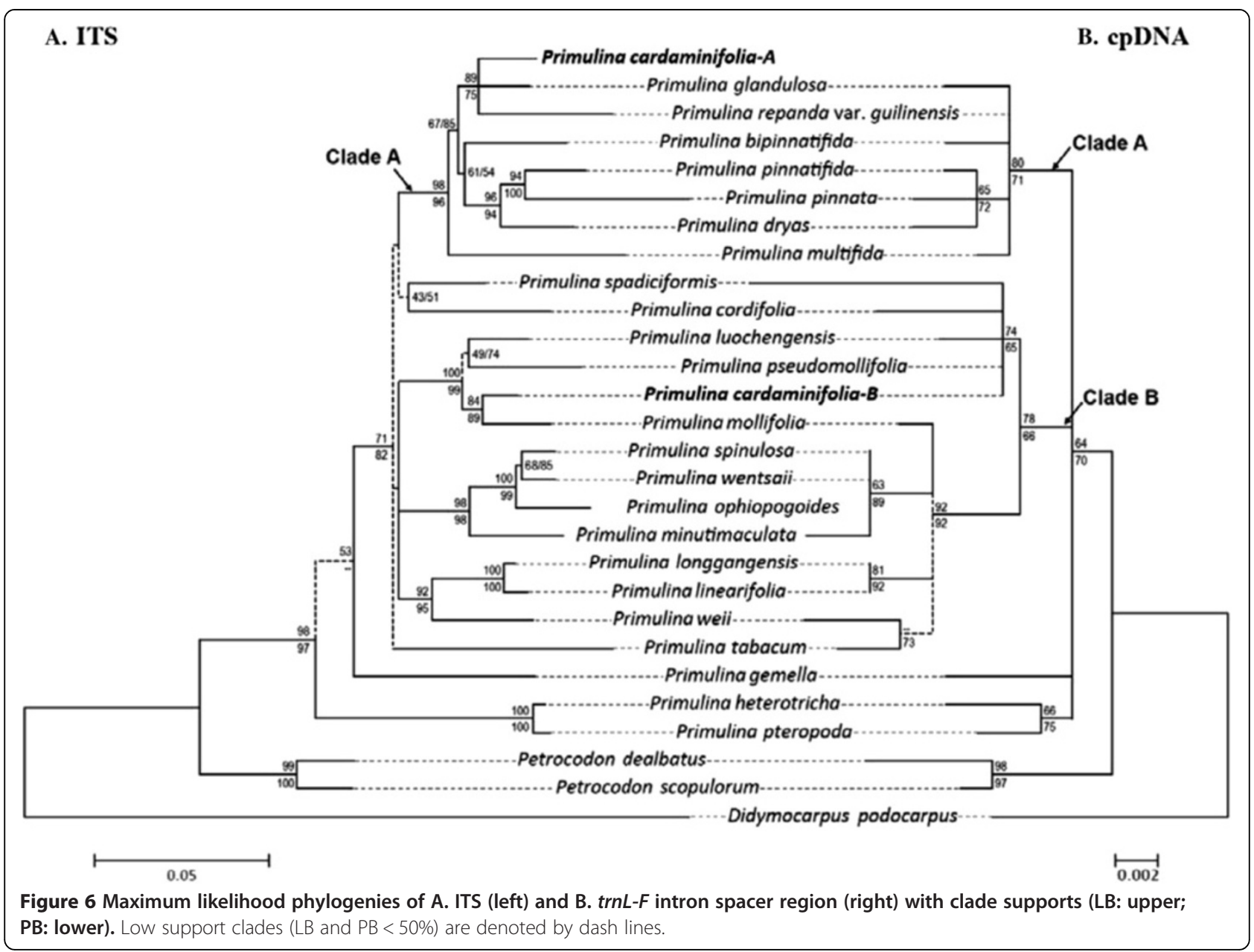

likelihood $=-4114.9869$ ) was recovered (Figure 6A). The MP analysis resulted in 6 equally parsimonious trees with 642 steps $(\mathrm{CI}=0.70, \mathrm{RI}=0.64, \mathrm{RCI}=0.44)$. The topology of the strict consensus tree of the 6 equally parsimonious trees was congruent with the ML tree (not shown). The cpDNA matrix contained 27 accessions of 694 aligned positions, of which only 19 (1.3\%) were parsimony informative. Based on the HasegawaKishino-Yano (HKY) selected by the AICc implemented in MEGA5, a single ML tree (log likelihood $=-1483.76)$ was obtained (Figure 6B). The MP analysis uncovered 23 equally parsimonious trees with 77 steps $(\mathrm{CI}=0.97$, $\mathrm{RI}=0.97, \mathrm{RCI}=0.94)$. The topology of the strict consensus tree of the 270 equally parsimonious trees was largely consistent with the ML tree (not shown).

Both ITS and cpDNA dataset placed $P$. cardaminifolia in Primulina with high $(\mathrm{LB}=98, \mathrm{~PB}=97)$ and low $(\mathrm{LB}=64$, $\mathrm{PB}=70)$ supports in ITS and cpDNA datasets, respectively (Figure 6), confirming its generic placement. Although the phylogenetic trees of the two datasets were not perfectly congruent with each other, especially in the deeper nodes, several well supported subclades were highly consistent between the two trees (Figure 6). Specifically, both datasets identified the clade (Clade A) composed of P. glandulosa, P. repanda var. guilinensis, P. bipinnatifida, P. dryas, $P$. pinnatifida, and P. multifida. In the ITS tree, the P. cardaminifolia-A sequence was also included in Clade A (Figure 6A). Other congruent clades included the clade consisting of $P$. spinulosa, $P$. wentsaii, $P$. ophiopogoides, and P. minutimaculata, the clade of $P$. longgangensis and $P$. linearifolia, and the clade of P. heterotricha and P. pteropoda.

The occurrence of intraindividual ITS polymorphism, or failure of concerted evolution among reiterated loci of ribosomal DNA arrays to nullify various rDNA repeats, could have resulted from hybridization, polyploidization, multiple nucelolar organizing regions on non-homologous chromosomes, rDNA pseudogenization, long generation time, loss of sexual recombination, or extensive introgression during domestication (Denduangboripant and Cronk 2000). The diploid chromosome number $2 n=36$ and the presence of two phylogenetically distinct ITS sequence types raised the concern that the distinctive P. cardaminifolia might have been a hybrid (e.g., Peng 


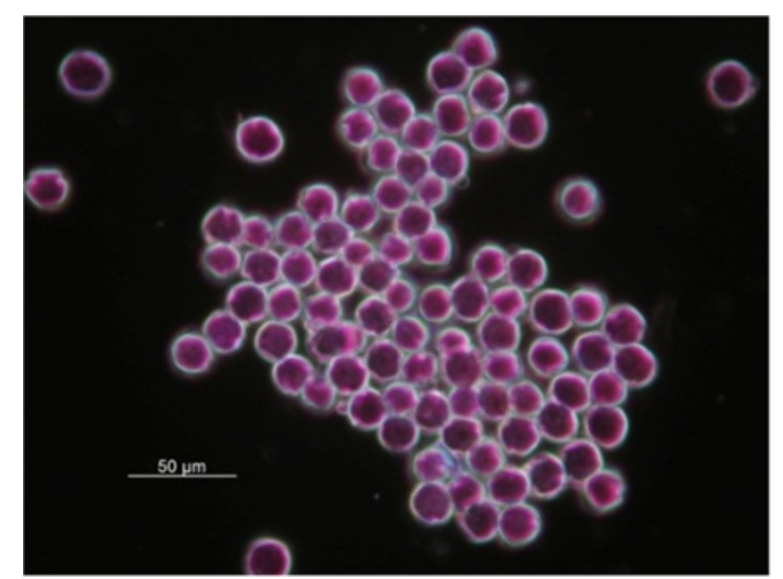

Figure 7 Microphotographs of pollen of Primulina cardaminfolia, showing normal and fully stained pollen.

and Chiang 2000), which has frequently been reported in Gesneriaceae (e.g., Puglisi et al. 2011). Assuming a maternal inheritance of its chloroplast genome, species in Clade B of the cpDNA tree would be the maternal parent, while Clade A could have been its paternal parent (Figure 6). Interestingly, most species in Clade A, including P. glandulosa, P. bipinnatifida, P. pinnatifidai, and $P$. multifida, are characterized by deeply lobed or pinnatified leaves that are otherwise unknown in Primulina and may have contributed to the unique imparipinnate leaves of $P$. cardaminifolia (Figures 1 \& 2). Nevertheless, the species status of $P$. cardaminifolia was supported by a $100 \%$ pollen fertility suggested by the high level of stainable pollen (Figure 7) using the malachite green-acid fuchsin-orange G stain (Alexander 1969). Given its perfectly developed pollen, the cytological and molecular data instead could have suggested that P. cardaminifolia might be of homoploid hybrid origin, which was formed without changes in chromosome number (cf. Howarth and Baum 2005; Rieseberg and Willis 2007; Abbott et al. 2010). Further studies will be needed to test the probable hybrid and homoploid origin of the new species.

\section{Conclusion}

All available data support the recognition of the new species Primulina cardaminifolia, which is described herein. The new species may have arisen via homoploid hybrid speciation. Its generic placement is confirmed by morphological, chromosomal, and molecular analyses.

\section{Appendix 1}

GenBank accession numbers: Species: (ITS/trnL-F). Pri mulina cardaminifolia was collected from the type locality [S.M. Ku 2035(HAST)].
Didymocarpus podocarpus C.B. Clarke: (DQ912688/ FJ501514); Petrocodon dealbatus Hance: (FJ501358/ FJ501537); Petrocodon scopulorum (Chun) YinZ. Wang [=Tengia scopulorum Chun]: (GU350637/GU350669); Primulina bipinnatifida (W.T. Wang) Yin Z. Wang [=Chiritopsis bipinnatifida W.T. Wang]: (DQ872842/ DQ872806); Primulina cardaminifolia Yan Liu \& W.B. Xu: (ITS-A: JX506738, ITS-B: JX506739/JX506740); Pri mulina cordifolia (D. Fang \& W.T. Wang) YinZ. Wang [=Chiritopsis cordifolia D. Fang \& W.T. Wang]: (DQ87 2845/DQ872803); Primulina dryas (Dunn) Mich. Möller \& A. Weber [=Chirita sinensis Lindl.]: (FJ501348/FJ5015 24); Primulina gemella (D. Wood) YinZ. Wang [=Chirita gemella D. Wood]: (FJ501345/FJ501523); Primulina glan dulosa (D. Fang, L. Zeng \& D.H. Qin) Yin Z. Wang [=Chiritopsis glandulosa D. Fang, L. Zeng \& D.H. Qin]: (DQ872841/DQ872804); Primulina heterotricha (Merr.) YinZ. Wang [=Chirita heterotricha Merr.]: (DQ872826/ DQ872816); Primulina linearifolia (W.T. Wang) YinZ. Wang [=Chirita linearifolia W.T. Wang]: (DQ872834/ DQ872810); Primulina longgangensis (W.T. Wang) YinZ. Wang [=Chirita longgangensis W.T. Wang]: (FJ501347/ AJ492290); Primulina luochengensis (Yan Liu \& W.B. Xu) Mich. Möller \& A. Weber [=Wentsaiboea luochengensis Yan Liu \& W.B. Xu]: (HQ633046/HQ632949); Primulina minutimaculata (D. Fang \& W.T. Wang) YinZ. Wang [=Chirita minutimaculata D. Fang \& W.T. Wang]: (DQ 872828/DQ872815); Primulina multifida B. Pan \& K.F. Chung: (JX507031/JX506756); Primulina ophiopogoides (D. Fang \& W.T. Wang) YinZ. Wang [=Chirita ophio pogoides D. Fang \& W.T. Wang]: (DQ872829/DQ872814); Primulina pinnata (W.T. Wang) YinZ. Wang [Chirita pinnata W.T. Wang]: (FJ501349/FJ501526); Primulina pin natifida (Hand.-Mazz.) Yin Z. Wang [=Chirita pinnatifida (Hand.-Mazz.) B.L. Burtt]: (FJ501350/FJ501527); Primu lina pseudomollifolia W.B. Xu \& Yan Liu: (JX506869/ JX506759); Primulina pteropoda (W. T. Wang) Yan Liu [=Chirita pteropoda W.T. Wang]: (DQ872827/DQ8728 17); Primulina repanda var. guilinensis (W.T. Wang) Mich. Möller \& A. Weber [=Chiritopsis repanda var. guilinensis W.T. Wang]: (DQ872846/DQ872808); Primu lina spadiciformis (W.T. Wang) Mich. Möller \& A. Weber [=Chirita spadiciformis W.T. Wang]: (FJ501346/AJ49 2291); Primulina spinulosa (D. Fang \& W.T. Wang) YinZ. Wang [=Chirita spinulosa D. Fang \& W.T. Wang]: (DQ8 72830/DQ872813); Primulina tabacum Hance: (FJ5013 52/AJ492300); Primulina weii Mich. Möller \& A. Weber [=Chirita mollifolia D. Fang, Y.G. Wei \& J. Murata]: (DQ872832/DQ872811); Primulina wentsaii (D. Fang \& L. Zeng) YinZ. Wang [=Chirita wentsaii D. Fang \& L. Zeng]: (DQ872831/DQ872812).

Competing interests

The authors declare that they have no competing interests. 


\section{Authors' contributions}

WBX and YL discovered the new species, WBX, YL, CIP, and KFC designed the project, YK collected the cytological data, and $\mathrm{HC}$ collected the molecular data. KFC performed the analyses and wrote the first draft of the manuscript. All authors read and approved the final manuscript.

\section{Acknowledgments}

The authors are grateful to Mr. Hong-Jin Wei for field assistance, Mr. Wen-Hong Lin (IBK) and Mr. Yun-Xi Zhu (IBK) for the high quality illustrations, Miss Hsun-An Yang for testing pollen stainability, and Dr. Michael Möller for critical review on the manuscript. This study was supported by the National Natural Science Foundation of China (Grant no. 41161011) and the Foundation of Key Laboratory of Plant Resources Conservation and Sustainable Utilization, South China Botanical Garden, Chinese Academy of Sciences to Yan Liu (IBK), and the National Geographic Society Grant \# 8358-07 to Ching-I Peng (HAST), Yan Liu (IBK) and Kuo-Fang Chung (NTUF).

\section{Author details}

${ }^{1}$ Guangxi Institute of Botany, Guangxi Zhuangzu Autonomous Region and the Chinese Academy of Sciences, Guilin 541006, China. ${ }^{2}$ Herbarium (HAST), Biodiversity Research Center, Academia Sinica, Nangang, Taipei 115, Taiwan. ${ }^{3}$ School of Forestry and Resource Conservation, National Taiwan University, Daan, Taipei 106, Taiwan.

\section{Received: 25 October 2012 Accepted: 27 February 2013} Published: 27 August 2013

\section{References}

Abbott RJ, Hegarty MJ, Hiscock SJ, Brennan AC (2010) Homoploid hybrid speciation in action. Taxon 59:1375-1386

Alexander MP (1969) Differential staining of aborted and nonaborted pollen. Biotech Histochem 44:117-122. doi:10.3109/10520296909063335

Christie F, Barber S, Möller M (2012) New chromosome counts in Old World Gesneriaceae: numbers for species hitherto regarded as Chirita, and their systematic and evolutionary significance. Edinburgh J Bot 69:323-345. doi:10.1017/S0960428612000169

Chung K-F, Huang H-Y, Peng C-I, Xu W-B (2013) Primulina mabaensis (Gesneriaceae), a new species from a limestone cave of northern Guangdong, China. Phytotaxa 92:40-48. doi:10.11646/phytotaxa.92.2.2

Denduangboripant J, Cronk QCB (2000) High intraindividual variation in internal transcibed spacer sequences in Aeschynanthus (Gesneriaceae): implications for phylogenetics. Proc Roy Soc Biol Sci Ser B 267:1407-1415. doi:10.1098/rspb.2000.1157

Hong X, Zhou S-B, Wen F (2012) Primulina chizhouensis sp. nov. (Gesneriaceae), a new species from a limestone cave in Anhui, China. Phytotaxa 50:13-18

Hou M-F, López-Pujol J, Qin H-N, Wang L-S, Liu Y (2010) Distribution pattern and conservation priorities for vascular plants in Southern China: Guangxi Province as a case study. Bot Stud 51:377-386

Howarth DG, Baum DA (2005) Genealogical evidence of homoploid hybrid speciation in an adaptive radiation of Scaevola (Goodeniaceae) in the Hawaiian Islands. Evolution 59:948-961. doi:10.1111/j.0014-3820.2005.tb01034.x

Huang Y-S, Xu W-B, Wu L, Liu Y (2012) Primulina gongchengensis (Gesneriaceae), a new species from Guangxi, China. Ann Bot Fenn 49:107-110. doi:10.5735/085.049.0118

Levan A, Fredga K, Sandberg AA (1964) Nomenclature for centromeric position on chromosomes. Hereditas 52:201-220. doi:10.1111/j.1601-5223.1964.tb01953.x

Li J, Wang Y, Hua G-J, Wen F (2012) Primulina xiziae sp. nov. (Gesneriaceae) from Zhejiang Province, China. Nord J Bot 30:77-81. doi:10.1111/j.17561051.2011.01185.x

Li J-M, Wang Y-Z (2007) Phylogenetic reconstruction among species of Chiritopsis and Chirita sect. Gibbosaccus (Gesneriaceae) based on nrDNA ITS and cpDNA trnL-F sequences. Syst Bot 32:888-898. http://www.bioone.org/doi/abs/ 10.1043/06-99.1?journalCode=sbot.

Li Z-Y, Wang Y-Z (2004) Plants of Gesneriaceae in China. Henan Science and Technology Publishing House. Zhengzhou, Henan

Liu R-R, Pan B, Zhou T-J, Liao J-P (2012) Cytological studies on Primulina taxa (Gesneriaceae) from limestone karsts in Guangxi province, China. Caryologia 65:295-303. doi:10.1080/00087114.2012.752920

Liu Y, Xu W-B, Huang Y-S (2011) Primulina guangxiensis sp. nov. (Gesneriaceae) from a karst cave in Guangxi, China. Nord J Bot 29:682-686. doi:10.1111/ j.1756-1051.2011.01089.x
Möller M, Forrest A, Wei Y-G, Weber A (2011) A molecular phylogenetic assessment of the advanced Asiatic and Malesian didymocarpoid Gesneriaceae with focus on non-monophyletic and monotypic genera. PI Syst Evol 292:223-248. doi:10.1007/s00606-010-0413-z

Möller M, Pfosser M, Jang C-G, Mayer V, Clark A, Hollingsworth ML, Barfuss MHJ, Wang Y-Z, Kiehn M, Weber A (2009) A preliminary phylogeny of the 'Didymocarpoid Gesneriaceae' based on three molecular data sets: incongruence with available tribal classifications. Am J Bot 96:989-1010. doi:10.3732/ajb.0800291

Peng C-I, Chiang T-Y (2000) Molecular confirmation of unidirectional hybridization in Begonia $\times$ taipeiensis Peng (Begoniaceae) from Taiwan. Ann Missouri Bot Gard 87:273-285

Puglisi C, Wei Y-G, Nishii K, Möller M (2011) Oreocharis $\times$ heterandra (Gesneriaceae): a natural hybrid from the Shengtangshan Mountains, Guangxi, China. Phytotaxa 38:1-18

Rieseberg LH, Willis JH (2007) Plant speciation. Science 317:910-914. doi:10.1126/ science.1137729

Tamura K, Peterson D, Peterson N, Stecher G, Nei M, Kumar S (2011) MEGA5: molecular evolutionary genetics analysis using maximum likelihood, evolutionary distance, and maximum parsimony methods. Mol Biol Evol 28:2731-2739. doi:10.1093/molbev/msr121

Wang W-T, Pan K-Y, Li Z-Y, Weitzman AL, Skog LE (1998) Gesneriaceae. In: Wu Z-Y , Raven PH (eds) Flora of China, vol 18. Science Press and Missouri Botanical Garden Press, Beijing and St, Louis, pp 244-401

Wang Y-Z, Mao R-B, Liu Y, Li J-M, Dong Y, Li Z-Y, Smith JF (2011) Phylogenetic reconstruction of Chirita and allies (Gesneriaceae) with taxonomic treatments. J Syst Evol 49:50-64. doi:10.1111/j.1759-6831.2010.00113.x

Weber A, Middleton DJ, Forrest A, Kiew R, Lim CL, Rafidah AR, Sontag S, Triboun P, Wei Y-G, Yao TL, Möller M (2011) Molecular systematics and remodelling of Chirita and associated genera (Gesneriaceae). Taxon 60:767-790

Wei Y-G (ed) (2010) Gesneriaceae of South China. Guangxi Science and Technology Publishing House, Guilin, Guangxi, 777 pp

Wen F, Wang F, Wei Y-G (2012a) Primulina yangshuoensis, a new species of Gesneriaceae from Guangxi, China. Taiwania 57:55-61

Wen F, Qin G-L, Wei Y-G, Liang G-Y, Gao B (2012b) Primulina hochiensis var. rosulata (Gesneriaceae) - a new variety at an entrance of a limestone cave from Guangxi, China. Phytotaxa 54:37-42

Wen F, Xi S-L, Wang Y, Xiang M-S, Fu L-F (2012C) Primulina fengshanensis (Gesneriaceae), a new species from Guangxi, China. Ann Bot Fenn 49:103-106. doi:10.5735/085.049.0117

Wu L, Zhang Q, Xu W-B, Mo S-S (2012a) Primulina guigangensis (Gesneriaceae): a new species from limestone area in Guangxi, China. Phytotaxa 38:19-23

Wu W-H, Meng T, Xu W-B, Liu S-Y, Zhang Q (2012b) Primulina sinovietnamica (Gesneriaceae), a new species identified by both morphological and molecular characters from the limestone area in Guangxi, China. Phytotaxa 60:32-40

Xu W-B, Pan B, Liu Y, Peng C-I, Chung K-F (2012a) Two new species, Primulina multifida and P. pseudomollifolia (Gesneriaceae), from karst caves in Guangxi, China. Bot Stud 53:165-175

Xu W-B, Zhang Q, Wen F, Liao W-B, Pan B, Chang H, Chung K-F (2012b) Nine new combinations and one new name of Primulina (Gesneriaceae) from South China. Phytotaxa 64:1-8

Yang X-Y, Liang K-M, Zhang X-H, Ma G-H (2012) Karyotype analysis of an endemic species Primulina tabacum (Gesneriaceae). PI Diversity Resources 34:25-27

\section{doi:10.1186/1999-3110-54-19}

Cite this article as: Xu et al:: Primulina cardaminifolia (Gesneriaceae), a rare new species from limestone areas in Guangxi, China. Botanical Studies 2013 54:19. 